\title{
Obtención de carburos de tantalio y de niobio por reducción carbotérmica del mineral columbotantalita
}

\author{
E. Gordo ${ }^{(*)}$, F. García-Carcedo $^{(*)}$ y J.M. Torralba ${ }^{(* *)}$
}

\begin{abstract}
Resumen Los carburos de tantalio y de niobio se caracterizan por su extremada dureza y resistencia al ataque químico. Ambos carburos, pero principalmente el de tantalio, se utilizan en la fabricación de metal duro (carburos cementados), junto con otros carburos, para herramientas de corte, matricería y para operaciones especiales de mecanizado que requieran resistencia al desgaste y resistencia al choque mecánico y a alta temperatura. También se está investigando su uso como refuerzo de aceros rápidos para el desarrollo de materiales resistentes al desgaste por vía pulvimetalúrgica. Sin embargo, el uso de $\mathrm{TaC}$ suele venir limitado por su alto precio, por lo que en ocasiones se utiliza carburo de tantalio con conteniendo de niobio, más barato. En el presente trabajo se estudia la obtención de carburos complejos de tantalio y de niobio a partir de un concentrado mineral de columbotantalita de procedencia española, mediante un proceso relativamente barato y sencillo, como es la reducción carbotérmica. Se describe el afino previo del mineral y su posterior reducción, así como la caracterización de los productos obtenidos.
\end{abstract}

Palabras clave: Carburos. Tantalio. Niobio. Tratamiento de menas.

\section{Tantalum and niobium carbides obtention by carbothermic reduction of columbotantalite ores}

\begin{abstract}
Tantalum and niobium carbides are characterized by its high hardness and chemical corrosion resistance. Both carbides, but mainly TaC, are used in hard metals (sintered carbides), together with other carbides, to manufacture cutting tools and dies and in special machining applications involving mechanical shock at high temperature. Its use as reinforcement of wear resistant materials through powder metallurgy techniques are being investigated. However, the use of $\mathrm{TaC}$ is usually limited because of its high cost. Therefore tantalum carbide with niobium content, which is cheaper, is used. In this work the obtention of complex tantalum and niobium carbides from a Spanish columbotantalite ore is studied through a relatively cheap and simple process as it is carbothermic reduction. Concentration of the ore, its reduction and the characterization of products are described.
\end{abstract}

Keywords: Carbides. Tantalum. Niobium. Ore treatment.

\section{INTRODUCCIÓN}

La columbotantalita es una de las menas principales del tantalio y del niobio, cuya fórmula general es $(\mathrm{Ta}, \mathrm{Nb})_{2}(\mathrm{Fe}, \mathrm{Mn}) \mathrm{O}_{6}(1)$. A partir de la columbotantalita se obtienen diversos productos, además de

(*) Centro Nacional de Investigaciones Metalúrgicas, CENIM (CSIC), Avda. de Gregorio del Amo, 8. 28040Madrid (España).

(**) Univ. Carlos III de Madrid. C/ Butarque, 15. 28911 Leganés (Madrid, España). los metales puros, tales como los pentóxidos $\left(\mathrm{Nb}_{2} \mathrm{O}_{5}, \mathrm{Ta}_{2} \mathrm{O}_{5}\right)$, o los carburos (NbC, TaC), mediante complejos sistemas que incluyen la extracción por disolventes y la separación líquidolíquido (2 y 3). El método comercial más utilizado para la producción de los carburos es la reducción carbotérmica de los pentóxidos puros (4 y 5). Sin embargo, se han realizado estudios encaminados a la obtención de los carburos mediante reducción directa del mineral columbotantalita (6 y 7), obteniéndose carburos complejos que podrían ser utilizados en lugar de mezclas de carburos puros, en algunas aplicaciones, como en los carburos 
cementados, o como refuerzo de aceros sinterizados, para mejorar su resistencia al desgaste e, incluso, su sinterabilidad (8).

Realmente, el proceso de reducción carbotérmica se utiliza para la obtención del metal, y se suele realizar en dos etapas, siendo el carburo el producto obtenido en la primera (9). El método se basa en la mayor afinidad de estos metales por el carbono que por el oxígeno a altas temperaturas y consiste, básicamente, en el calentamiento de una mezcla de óxido con la cantidad estequiométrica de carbono para que se produzcan las reacciones en un horno con atmósfera inerte o reductora. Para establecer las condiciones de operación, son útiles los diagramas de cambio de energía libre $(\Delta G)$ en función de la temperatura para las reacciones que intervienen en la reacción global de reducción. En este sentido, si consideramos la columbotantalita idealmente compuesta por una mezcla de óxidos $\mathrm{Nb}_{2} \mathrm{O}_{5}, \mathrm{Ta}_{2} \mathrm{O}_{5}$, $\mathrm{FeO}$ y $\mathrm{MnO}$, las reacciones más probables de estos óxidos con el carbono y el cambio de energía libre en función de la temperatura, se muestran en la figura 1, en la que se observa que desde el punto de vista termodinámico $\mathrm{y}$, a partir de $1.450{ }^{\circ} \mathrm{C}$, todas las reacciones planteadas son posibles.

Sin embargo, la reducción de la columbotantalita con carbono, puede sufrir variaciones respecto a la reducción de los óxidos puros en los que puede descomponerse dicho mineral, ya que se encuentran presentes otros elementos y compuestos en forma de impurezas, que pueden dar lugar a la formación de compuestos intermedios difíciles de determinar. Es necesario, por tanto, partir de un concentrado de columbotantalita lo más puro posible. En el presente trabajo se describen los métodos utilizados para eliminación de impurezas previamente a la realización de los ensayos de reducción carbotérmica, métodos también utilizados comercialmente en algunas plantas de extracción de estos minerales.

\section{PROCEDIMIENTO EXPERIMENTAL}

El material de partida es un concentrado mineral de columbotantalita procedente de la mina de Penouta (Orense, España), obtenido como subproducto del beneficio del mineral de casiterita, cuya composición química se recoge en la tabla I.

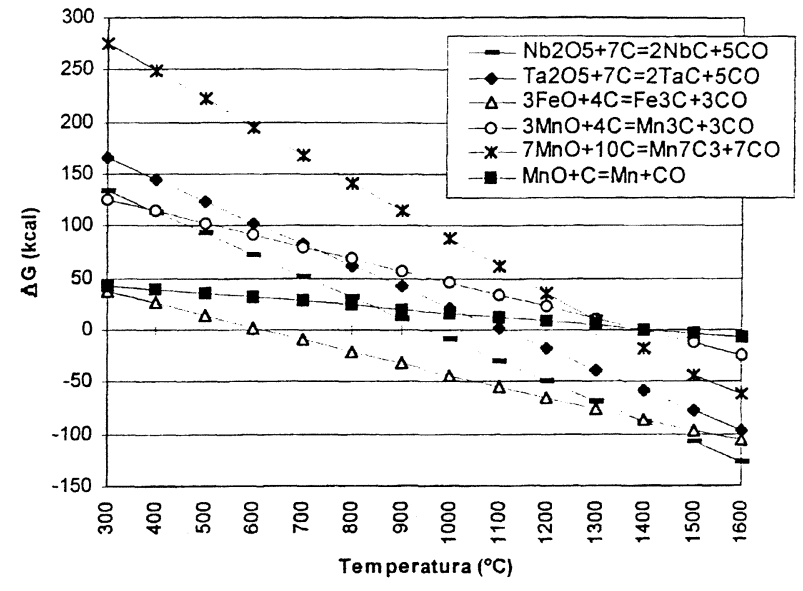

FIG. 1.- Cambio de energía libre en las reacciones de reducción de la columbotantalita.

FIG. 1.- Free energy change in columbotantalite reduction.

Aprovechando el carácter conductor y ligeramente magnético de la columbotantalita (10) se utilizaron la separación magnética y la separación electrostática como medios físicos para la eliminación de las impurezas que acompañan al mineral, como casiterita, ilmenita, cuarzo, topacio, mica, feldespatos, granates y otros silicoaluminatos. Los equipos utilizados en dichas separaciones fueron un separador magnético de alta intensidad Franz y un separador electrostático Boxmag Rapid tipo HT 150. Los concentrados obtenidos después de las sucesivas etapas de separación fueron caracterizados mediante diversas técnicas: análisis químico, fluorescencia de rayos X (FRX), difracción de rayos $\mathrm{X}$ (DRX), microscopía electrónica de barrido (MEB), microanálisis por dispersión de energías (EDX) y análisis granulométricos. Posteriormente, se sometieron a procesos de lixiviación en ácido sulfúrico, a temperatura de ebullición y con agitación, para eliminar impurezas ricas en manganeso que no habían podido ser eliminadas por medios físicos; de nuevo, se caracterizó el concentrado obtenido. Este producto fue sometido al proceso de carburización por reducción carbotérmica, utilizando las condiciones establecidas en trabajos anteriores, a saber: temperatura, $1.550^{\circ} \mathrm{C}$; atmósfera de nitrógeno $\mathrm{N} 45$; tiempo, 5 h; relación mineral/carbono, 6,25. Los ensayos se realizaron en un horno

TABLA I.- Composición química del concentrado de columbotantalita inicial, \% en masa

TABLE I.-Chemical composition of the initial columbotantalite concentrate, \% in mass

\begin{tabular}{|c|c|c|c|c|c|c|c|c|c|c|c|}
\hline \multicolumn{10}{|c|}{ Análisis químico } \\
\hline $\mathrm{Ta}$ & $\mathrm{Nb}$ & $\mathrm{Mn}$ & $\mathrm{Fe}$ & $\mathrm{Si}$ & $\mathrm{Al}$ & $\mathrm{Sn}$ & $\mathrm{P}$ & $\mathrm{K}$ & $\mathrm{Ti}$ & $\mathrm{Zn}$ & $\mathrm{Cu}$ \\
\hline 23,22 & 19,78 & 11,9 & 3,21 & 1,2 & 0,56 & 0,83 & 0,25 & 0,21 & 0,14 & 0,12 & 0,037 \\
\hline
\end{tabular}


tubular vertical con control de atmósfera. Los productos resultantes se caracterizaron utilizando las técnicas mencionadas, además de la medida del contenido de oxígeno mediante un equipo Leco TC436. La figura 2 muestra las etapas en las que se ha llevado a cabo el proceso experimental.

\section{RESULTADOS Y DISCUSIÓN}

Las etapas de concentración destinadas a la eliminación de las impurezas dieron lugar a concentrados cuya composición química, expresada en forma de óxidos, se recoge en la tabla II.

El mayor porcentaje de pentóxidos de tantalio y de niobio en el concentrado obtenido por medios físicos se debe, fundamentalmente, a la eliminación de silicoaluminatos y feldespatos de potasio. Esta afirmación se basa en la caracterización de los concentrados mediante FRX, MEB y EDX, que han permitido realizar un seguimiento de la progresiva eliminación de impurezas. Mediante FRX, se realizó el análisis químico semicuantitativo de las fracciones obtenidas en las distintas etapas de separación por medios físicos. Mediante MEB se obtuvieron imágenes de electrones retrodispersados (BSE) en las que se identificaron las diferentes fases, cuya composición se obtuvo por EDX (Fig. 3).

Los productos resultantes de los ensayos de carburización tienen la composición química que se muestra en la tabla III, y presentan espectros DRX como el de la figura 4.

Además, las imágenes obtenidas mediante MEB (Fig. 5) permiten distinguir zonas cristalizadas en forma de cubos y zonas de aspecto amorfo, que hacen de aglomerante o recubrimiento de los cubos.

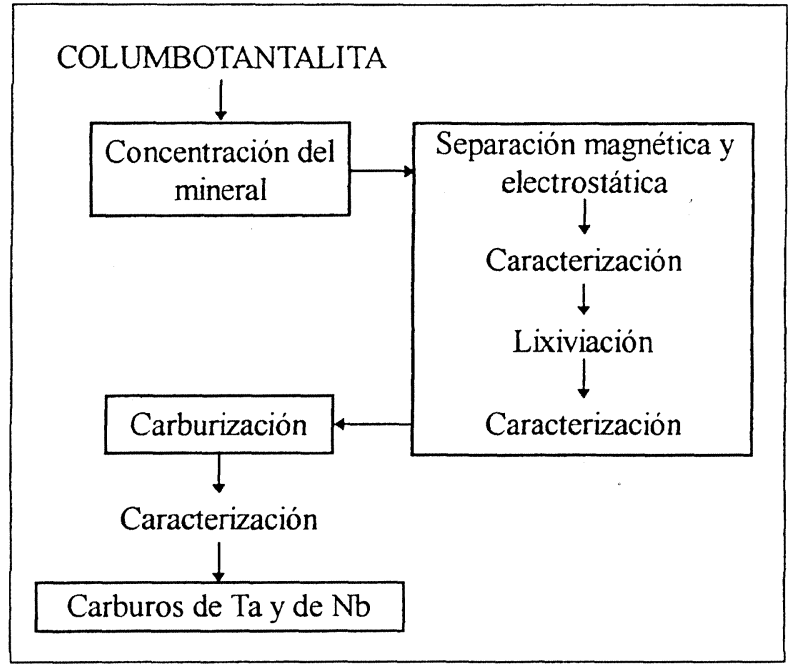

FIG. 2.- Etapas del procedimiento experimental.

FIG. 2.- Steps of the experimental procedure.
TABLA II.- Composición química de los productos obtenidos en las etapas de concentración, \% en masa

TABLE II.- Chemical composition of the products obtained in the concentration steps, \% in mass

\begin{tabular}{|c|c|c|c|c|c|}
\hline Material & $\mathrm{Nb}_{2} \mathrm{O}_{5}$ & $\mathrm{Ta}_{2} \mathrm{O}_{5}$ & $\mathrm{FeO}$ & $\mathrm{MnO}$ & Total \\
\hline $\begin{array}{c}\text { Concentrado } \\
\text { inicial }\end{array}$ & 28,29 & 28,35 & 4,13 & 15,36 & 76,13 \\
\hline $\begin{array}{c}\text { Concentrado } \\
\text { obtenido por } \\
\text { medios físicos }\end{array}$ & 32,9 & 30,04 & 4,0 & 17,04 & 83,98 \\
\hline $\begin{array}{c}\text { Concentrado } \\
\text { obtenido por } \\
\text { lixiviación }\end{array}$ & 39,34 & 35,29 & 4,03 & 10,71 & 89,37 \\
\hline
\end{tabular}
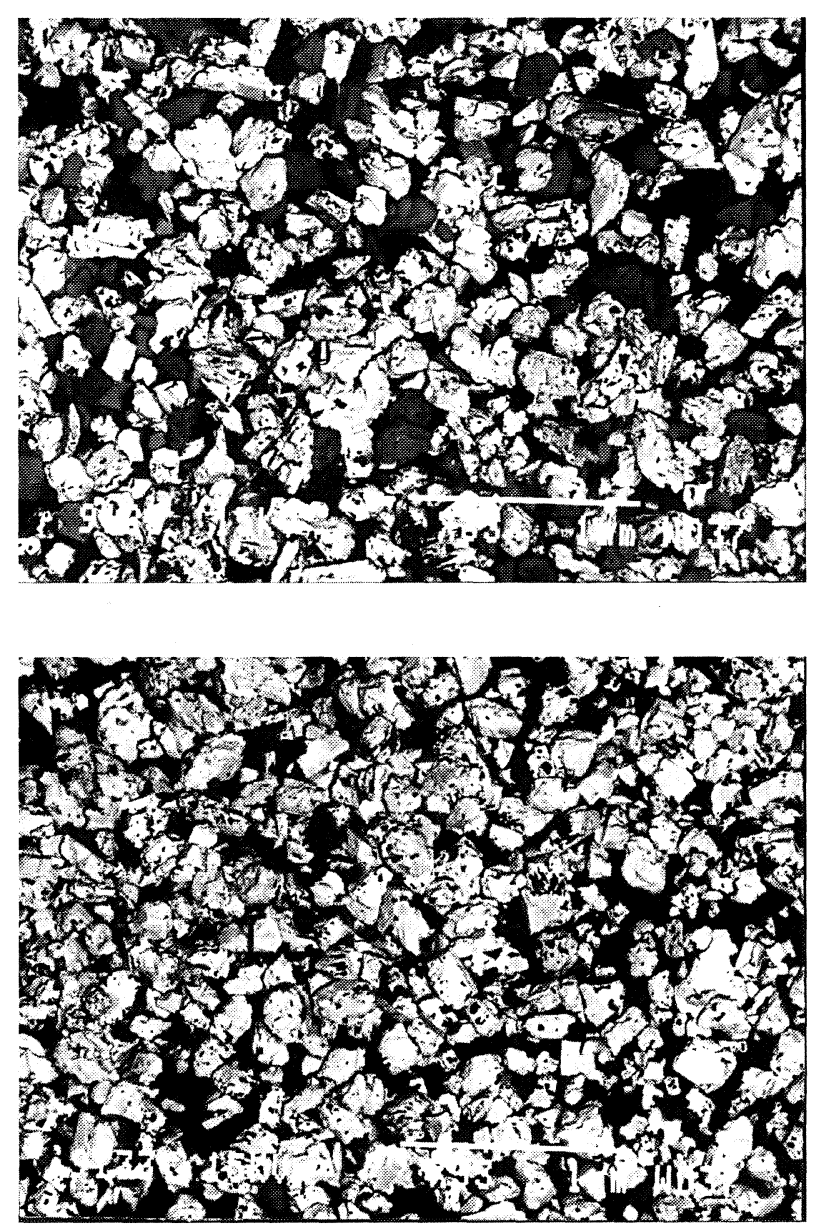

FIG. 3.- Imagen BSE del concentrado obtenido por: $a$ ) medios físicos. $b$ ) por lixiviación.

FIG. 3.-BSE image from concentrate obtained by: a) physical methods. b) leaching.

El microanálisis por EDX de ambas zonas revela que los cubos están formados por tantalio y niobio, mientras que las zonas amorfas son ricas en manganeso y hierro. Teniendo en cuenta que, tanto los carburos de niobio (NbC) como los de tantalio 
TABLA III.- Composición química de los productos de los ensayos de carburización, \% en masa

TABLE III.-Chemical composition of the carburization products, \% in mass

\begin{tabular}{|c|c|c|c|c|c|c|c|c|}
\hline \multicolumn{7}{|c|}{ Elementos } \\
\hline $\mathrm{Nb}$ & $\mathrm{Ta}$ & $\mathrm{Mn}$ & $\mathrm{Fe}$ & $\mathrm{Si}$ & $\mathrm{Al}$ & $\mathrm{Si}$ & $\mathrm{C}$ & $\mathrm{O}$ \\
\hline 36,9 & 34,03 & 10,2 & 5,55 & 1,93 & 0,58 & 0,9 & 6,2 & 3,4 \\
\hline \multicolumn{7}{|c|}{ Compuestos más probables } \\
\hline $\mathrm{NbC}$ & $\mathrm{TaC}$ & $\mathrm{Fe}_{3} \mathrm{C}$ & $\mathrm{Mn}_{3} \mathrm{C}$ & $\mathrm{MnO}$ & $\mathrm{SiO}_{2}$ & $\mathrm{Al}_{2} \mathrm{O}_{3}$ \\
\hline 41,6 & 36,3 & 5,9 & 4,16 & & 8,2 & 1,93 & 1,11 \\
\hline \multicolumn{7}{|c|}{ Total carburos: 87,96} & \multicolumn{5}{c|}{ Total óxidos: 11,24} \\
\hline
\end{tabular}

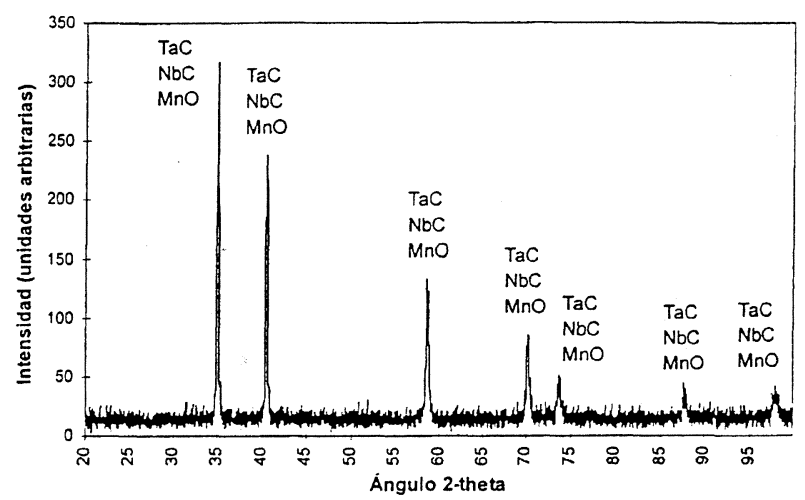

FIG. 4.- Espectro DRX de los productos de los ensayos de carburización.

FIG. 4.- DRX spectrum of the carburization products.

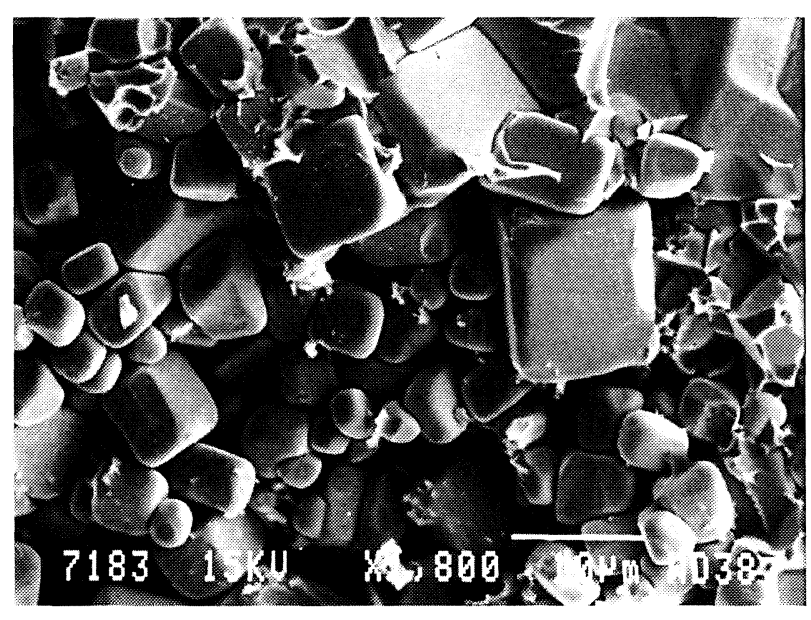

FIG. 5.- Imagen SEM del producto obtenido por carburización.

FIG. 5.- SEM image obtained from products obtained by carburization.

(TaC), cristalizan en el sistema cúbico centrado en las caras (11), y los resultados obtenidos en el análi- sis por DRX, se puede afirmar que los cristales cúbicos son carburos de tantalio y de niobio o mixtos, siendo esta opción la más probable dado que el microanálisis por EDX de las zonas cristalizadas muestra invariablemente la presencia de ambos elementos.

El análisis por DRX indica también la presencia en la muestra de $\mathrm{MnO}$. Puesto que el detector de dispersión de energías acoplado al MEB utilizado en este estudio, no permite detectar elementos ligeros, como el carbono o el oxígeno, se prepararon muestras embutidas en resina y pulidas, y se realizaron mapas de rayos $\mathrm{X}$ de las líneas $\mathrm{K} \alpha$ del manganeso y del oxígeno de algunas zonas en las que se distinguían fases ricas en manganeso, y fases ricas en manganeso y silicio. El resultado de este estudio indicaba que las zonas más ligeras (constituidas por manganeso y silicio) también contenían oxígeno, mientras que las zonas en las que únicamente se encontraba manganeso, no aparecía oxígeno. Esto parece apuntar a que parte del manganeso se encuentra en forma de óxido y parte en forma metálica o de carburo. Los datos termodinámicos (Fig. 1) indican que, a la temperatura a la que se han llevado a cabo los ensayos de carburización (1.550 ${ }^{\circ} \mathrm{C}$ ), es posible la reducción del $\mathrm{MnO}$ a manganeso, si bien son más favorables las reacciones que dan lugar a la formación de carburos. Por otro lado, el hierro se encontrará probablemente en forma de carburo, y el silicio y el aluminio, en forma de óxidos, aunque no se han detectado estos compuestos mediante DRX.

Teniendo en cuenta las consideraciones realizadas, se puede hacer un reparto del carbono y del oxígeno contenidos en el producto carburado, entre los distintos elementos mayoritarios, quedando la composición en forma de elementos más probables señalada en la tabla III. Esta tabla indica que se ha obtenido un producto con un $78 \%$ en masa de carburos de $\mathrm{NbC}+\mathrm{TaC}$, que asciende a un $88 \%$ si se suman los carburos de hierro y manganeso. Sin 
embargo, la cantidad de óxidos presentes resulta alta, siendo necesaria una etapa de lixiviación posterior a la carburación para eliminar, al menos, los óxidos de manganeso.

\section{CONCLUSIONES}

Este trabajo sienta las bases para la obtención, mediante un método sencillo y relativamente barato, de carburos mixtos de tantalio y de niobio, que permite obviar las costosas etapas de separación de los dos metales.

Los métodos utilizados para la concentración del mineral inicial han resultado exitosos, revelándose el proceso de lixiviación como fundamental para la eliminación de los óxidos de manganeso, y como necesario paso posterior a la carburización para eliminar los óxidos de manganeso que acompañan a los carburos. Por su parte, la carburización por reducción directa del concentrado obtenido ha dado lugar a un producto con un porcentaje de carburos de tantalio y de niobio que alcanza el $78 \%$ en masa del total, pero susceptible de ser obtenido con mayor pureza.

\section{Agradecimiento}

Este trabajo ha sido posible gracias a la financiación por la CICYT del proyecto MAT96-0542, ya finalizado, y del proyecto MAT97-0695, actualmente en curso, y a los trabajos realizados con anterioridad por el Dr. J. C. Ruiz Sierra ( $\dagger$ ), iniciador e impulsor de ambos proyectos y de esta línea de investigación.

\section{REFERENCIAS}

(1) Rohmer, R. y Foex, M. Nouveau Traité de Chimie Minérale, de Paul Pascal. Masson et Cie, Éditeurs. Tomo XII. París $1958: 281$.

(2) LAmbert, B. y Droegkamp, R.E. Production of tantalum and niobium powders. Metals Handbook, American Society for Metals. Vol. 7. $9^{a}$ edi. (ASM). 1984 : 160-163.

(3) Rockenbauer, W. Proc. of the Int. Symp. Niobium ' 81. San Francisco (Calif., EE.UU.) 8-11 Nov. 1981 : 133-152.

(4) SURI, A.K. y GuPTA, C.K. Trans. Indian Inst. Metals, 31 (2), $1978: 152-156$.

(5) Garg, S.P., Venkataramani, R. y Sundaram, C.V. Trans. Indian Inst. Metals, 28 (4), 1975 : 281-288.

(6) Ono, K. High Temp. Mater. Proc. 11 (1-4), 1993 : 207216.

(7) Gaballah, I., Meyer-Joly, M., Djona, M., Allain, E., Kanari, N., Ruiz, J.C., Chejne, F., Fernandez, M., Ayala, N., Garcia, F. "Recovery of Ta and Nb from concentrates and by-products of European ores type Echassières (France) and Penouta (Spain)". Report 4. Contract MA1M-0071-C(CD) European Community. 08/1990.

(8) GoRDo, E. Refuerzo de aceros rápidos por adición de carburos complejos de $\mathrm{Ta}$ y de $\mathrm{Nb}$. Tesis Doctoral. Univ. Politécnica de Madrid, 1998.

(9) Kolchin, O.P. Soviet J. Non-Ferrous Met. 11 (julio), $1970: 49-51$.

(10) Catálogo CARPCO/DENVER, 1954.

(11) SCHWARZKOPF, P. y KIEFFER, R. Refractory hard metals. Borides, carbides, nitrides and silicides. The Macmillan Company. Nueva York, 1953. 